\title{
Prevalence and spatial distribution patterns of human echinococcosis at the township level in Sichuan Province, China
}

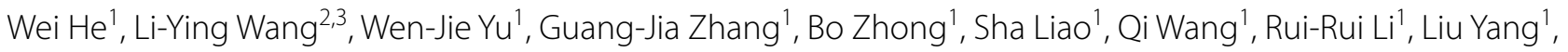
Ren-Xin Yao ${ }^{1}$, Yang Liu' ${ }^{1}$ Zeli Danba ${ }^{4}$, Sheng-Chao Qin ${ }^{5}$, Shi-An Wang ${ }^{6}$, Yan-Xia Wang ${ }^{7}$, Yan Huang ${ }^{1 *}$ and Qian Wang ${ }^{1^{*}}$ (1)

\begin{abstract}
Background: Echinococcosis is a global zoonotic parasitic disease caused by Echinococcus larvae. This disease is highly endemic in Sichuan Province, China. This study investigates the prevalence and spatial distribution characteristics of human echinococcosis at the township level in Sichuan Province, geared towards providing a future reference for the development of precise prevention and control strategies.

Methods: Human prevalence of echinococcosis was evaluated using the B-ultrasonography diagnostic method in Sichuan Province between 2016 and 2019. All data were collected, collated, and analyzed. A spatial distribution map was drawn to intuitively analyze the spatial distribution features. Eventually, the spatial autocorrelation was specified and local indicators of spatial association (LISA) clustering map was drawn to investigate the spatial aggregation of echinococcosis at the township level in Sichuan Province.

Results: The prevalence of echinococcosis in humans of Sichuan Province was $0.462 \%$, among which the occurrence of cystic echinococcosis (CE) was $0.221 \%$, while that of alveolar echinococcosis (AE) was $0.244 \%$. Based on the results of the spatial distribution map, a predominance of echinococcosis in humans decreased gradually from west to east and from north to south. The Global Moran's / index was $0.77(Z=32.07, P<0.05)$, indicating that the prevalence of echinococcosis in humans was spatially clustered, exhibiting a significant spatial positive correlation. Further, the findings of local spatial autocorrelation analysis revealed that the "high-high" concentration areas were primarily located in some townships in the northwest of Sichuan Province. However, the "low-low" concentration areas were predominantly located in some townships in the southeast of Sichuan Province.
\end{abstract}

Conclusions: Our findings demonstrated that the prevalence of echinococcosis in humans of Sichuan Province follows a downward trend, suggesting that the current prevention and control work has achieved substantial outcomes. Nevertheless, the prevalence in humans at the township level is widely distributed and differs significantly, with a clear clustering in space. Therefore, precise prevention and control strategies should be formulated for clusters, specifically strengthening the "high-high" clusters at the township level.

Keywords: Echinococcosis, Prevalence, Spatial autocorrelation, Sichuan, China

*Correspondence: 1308700501@qq.com; wangqian1967@163.com ${ }^{1}$ Department of Parasitic Diseases, Sichuan Provincial Center for Disease Control and Prevention, No.6 Zhongxue Road, Chengdu 610041, People's Republic of China

Full list of author information is available at the end of the article

\section{Background}

Echinococcosis (also known as hydatid disease) is a zoonotic parasitic disease caused by the larvae of Echinococcus spp. It belongs to group $\mathrm{C}$ infectious diseases 
in China due to its specific manifestation and transmission mode in hosts. Due to its severity, echinococcosis is a global public health problem [1-3]. Previous studies reported that four species of the genus Echinococcus, including E. granulosus, E. multilocularis, E. oligarthrus, and $E$. vogeli, are responsible for echinococcosis in humans; and E. granulosus and E. multilocularis are the most significant $[4,5]$. Larvae of these four species are subjected to three types of echinococcosis in humans, including cystic echinococcosis (CE), alveolar echinococcosis (AE), and polycystic echinococcosis (PE) [6]. Infected canines and felines including dogs, wolves, foxes, and cats are important infection sources of echinococcosis. Intermediate hosts include even-toed ungulates and small mammals, while humans are implicated as incidental hosts. This disease is primarily transmitted via the fecal-oral route. Individuals living in echinococcosis-endemic areas are susceptible to echinococcosis, and high-risk groups primarily include those involved in agricultural production, livestock slaughter, fur processing, and hunting. The life cycle of Echinococcus spp. must be completed by canine (feline) and cloven-hoofed animal hosts/small mammals, where three processes including egg, metacestode, and adult worm are involved. This disease has clinically characterized symptoms, including cyst pressure and irritation to affected organs, pain, fever, and allergic reactions [7]. Studies indicate that the mortality rate of $\mathrm{AE}$ exceeds $90 \%$ in patients either untreated or undertreated for 10-15 years [8].

Echinococcosis is globally widespread, showing priority in agricultural and pastoral areas. Different Echinococcus spp. causing different types of echinococcosis exhibits different global distributions, causing differences in the prevalence of different types of echinococcosis and regions. Only two types of echinococcosis (CE and $\mathrm{AE}$ ) have been reported in China. The global disease burden of AE in terms of disability-adjusted of life years (DALY) in China accounts for 91\%, while that of CE accounts for $40 \%[9,10]$. Multiple recent reports indicate that echinococcosis is primarily dominant in pastoral, semi-agricultural, and semi-pastoral areas of Inner Mongolia, Sichuan, Tibet, Gansu, Qinghai, Ningxia, Yunnan, Shaanxi, and Xinjiang provinces/autonomous regions in northwest China, specifically in Sichuan, Tibet, and Qinghai in Qinghai-Tibet Plateau [11, 12]. Of these regions, Sichuan is one of the provinces with the most severe prevalence of echinococcosis in China, with a mixed epidemic area of $\mathrm{CE}$ and $\mathrm{AE}$. Based on a report by the national survey conducted in 2012, echinococcosis was prevalent in 35 counties in Sichuan, mainly predominant in the whole area of Ganzi (18 counties) and Aba prefectures (13 counties), in Muli and Yuexi counties of Liangshan prefecture, also in Tianquan and Baoxing counties of Ya'an city. Subsequently, the estimated prevalence was $1.08 \%[13,14]$.

Although the occurrence of echinococcosis in Sichuan Province is clear at the county level [14], it remains elusive at the township level and warrants further inquiry. Herein, we performed echinococcosis screening for the whole population in Sichuan Province between 2016 and 2019 to unravel the current status of the human echinococcosis prevalence.

The spatial statistical analysis method has been widely applied in the field of epidemiologic study of echinococcosis, specifically in evaluating the difference of prevalence in different regions and identifying disease clustering $[15,16]$. For instance, Brundu et al. analyzed the spatial scan statistics of 1029 pastures where CE of bovine was discovered in Sardinia and Italy, revealing two clusters [17]. Elsewhere, a spatial autocorrelation analysis of echinococcosis prevalence in 13 counties of Aba Prefecture in Sichuan Province was conducted using the global Moran's I method by Qi demonstrating significant clustering distribution in echinococcosis prevalence. However, the global Getis'G result elucidated clusters with a high prevalence of echinococcosis [18]. Additionally, Zhao et al. used spatial scan statistics and spatial autocorrelation analysis methods to assess the detection rate of echinococcosis in 18 counties of Ganzi prefecture in Sichuan Province, and reported aggregation in the spatial distribution of echinococcosis [19].

This study analyzed the spatial aggregation of human echinococcosis prevalence at the township level in Sichuan Province using global and local spatial autocorrelation analysis methods. This was geared towards identifying spatial aggregation areas of echinococcosis at the township level in Sichuan Province, to establish the areas that should strengthen the key prevention and control in the future. Besides, we purposed to provide a baseline reference for the formulation of precise strategies and measures for the prevention and mitigation of echinococcosis.

\section{Methods}

\section{Survey areas}

A survey conducted between 2016 and 2019 was used to investigate the prevalence of human echinococcosis in areas where many echinococcosis cases were previously reported. The scope of this study was full coverage comprising 325 townships under 18 counties in Ganzi prefecture, 230 townships under 13 counties in Aba prefecture, 70 townships under 2 counties in Liangshan prefecture, and 24 townships under two counties in Ya'an city. Overall, 649 townships were covered to screen human echinococcosis (Fig. 1). Among the 35 endemic counties of echinococcosis in Sichuan 


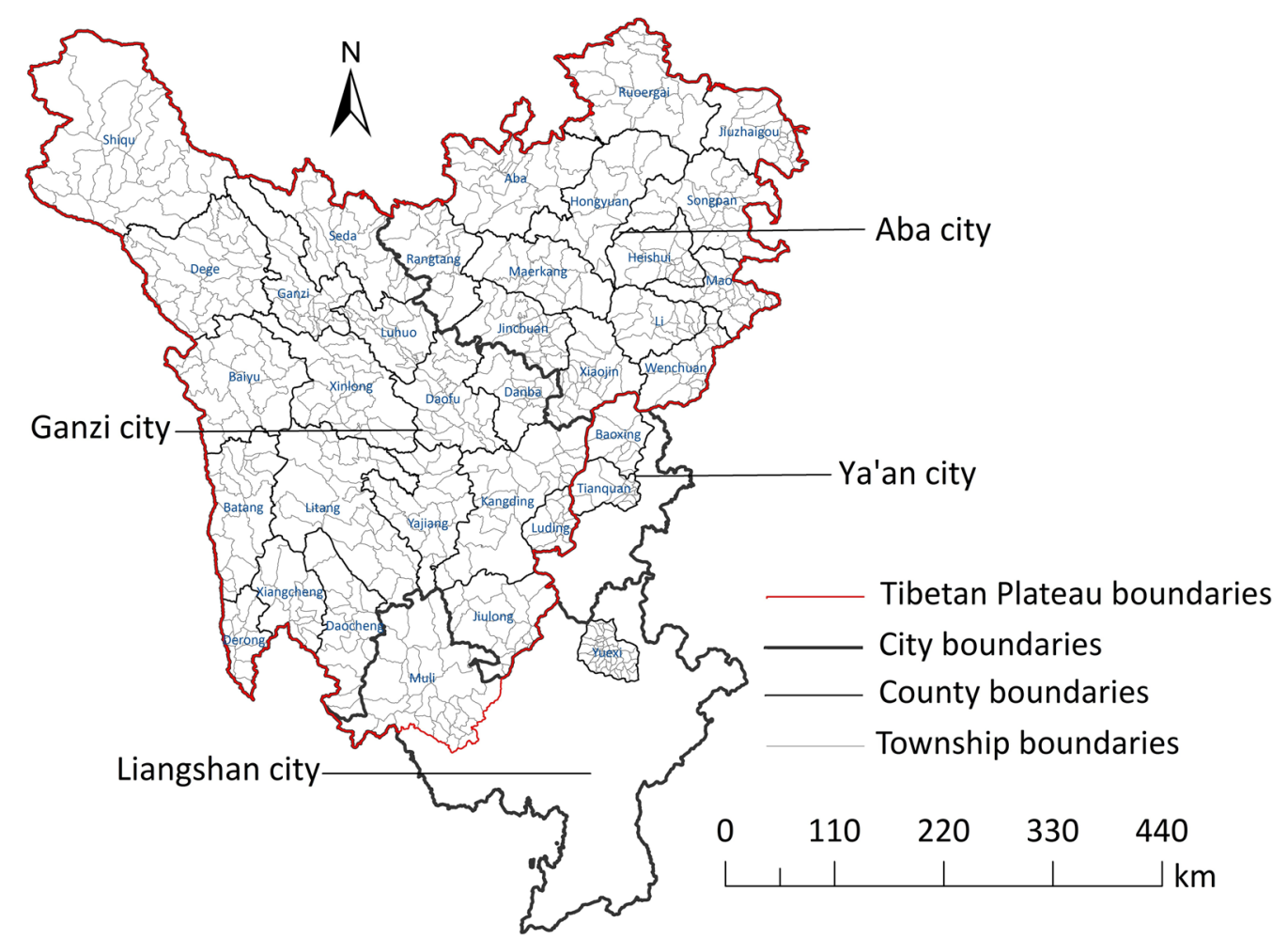

Fig. 1 Map of townships for echinococcosis screening in Sichuan Province

Province, except Tianquan, Baoxing, and Yuexi counties, the rest counties belong to the Qinghai-Tibet Plateau.

\section{Survey contents and methods Target population}

All permanent residents (including those who had a continuous residence at the survey areas for more than 6 months) aged 2 years and above were screened for echinococcosis.

\section{Survey of prevalence}

The prevalence of human echinococcosis was investigated based on the requirements of the Technical Plan for Echinococcosis Prevention and Control [20]. A B-ultrasound examination of the abdomen was performed using a portable ultrasonic diagnostic instrument. Patients were diagnosed following the Diagnostic Criteria for Echinococcosis (WS257-2006) [21], while serological tests were supplemented to the suspected patients. Specifically, the anti-echinococcosis antibody in the serum of suspected patients was detected using ELISA. The IgG Antibody Diagnostic Kit was purchased from Shenzhen Kangbaide Biotechnology Co., Ltd., China.

\section{Spatial clustering analysis}

The detection rate of human echinococcosis in Sichuan Province was analyzed by global autocorrelation and local control autocorrelation to understand the aggregation degree and scope of echinococcosis. Based on the literature, spatial autocorrelation accurately reflected the aggregation degree of an indicator in a spatial unit. Spatial autocorrelation analysis included global spatial autocorrelation and local spatial autocorrelation analyses. Spatial autocorrelation coefficients are often used to quantitatively judge the spatial autocorrelation form and correlation size. Several spatial autocorrelation coefficients suitable for different data types have been reported. For instance, the statistics of common adjacent edges are suitable for type variables, while the statistics of Moran's $I$, Geary's $C$, and Getis $G$ are primarily suitable for numerical variables. Since the prevalence of echinococcosis was a numerical variable, Moran's I statistics were used for analysis [22].

\section{Global spatial autocorrelation analysis}

Global spatial autocorrelation analysis was used to analyze whether the specified attributes in the whole research scope exhibit autocorrelation and global 
Moran's $I$ index can be used for global spatial autocorrelation analysis, and the formula is as follows:

$$
I=\frac{n \sum_{i=1}^{n} \sum_{j=1}^{n} w_{i j}\left(x_{i}-\bar{x}\right)\left(x_{j}-\bar{x}\right)}{\sum_{i=1}^{n} \sum_{j=1}^{n} w_{i j} \sum_{i=1}^{n}\left(x_{i}-\vec{x}\right)^{2}}
$$

where $n$ refers to the total number of observed values, and $x_{i}$ refers to the observed value at position $i . x_{j}$ is the observed value at position $j, i \neq j . \bar{x}$ refers to the average of observed values at all $n$ positions, $\bar{x}=\frac{1}{n} \sum_{i=1}^{n} x_{i} \cdot w_{i j}$ refers to the element value of symmetric binomial distribution spatial weight matrix, a measure of the influence and action between spatial positions $i$ and $j$.

Moran's $I$ range from -1 to +1 . Moran's $I$ index greater than 0 indicates that $x_{i}$ and $x_{j}$ change in the same direction, and the data show positive autocorrelation. The closer the value was to +1 , it indicated that similar observed values gather in a similar region, and the stronger the positive correlation, the higher the regional aggregation. Moran's $I$ index was less than 0 , suggesting that $x_{i}$ and $x_{j}$ change in different directions and the data were negatively correlated. The closer the value was to -1 , the stronger the negative correlation when different observed values were gathered; Moran's $I$ index was close to 0 , indicating that the observed values were mostly randomly distributed in space with no spatial autocorrelation.

Global spatial autocorrelation analysis was used to establish whether the prevalence of echinococcosis exists aggregation in the whole space of Sichuan Province. Only with aggregation in the whole space, local spatial autocorrelation analysis was carried out.

\section{Local spatial autocorrelation analysis}

Local spatial autocorrelation analysis was used to analyze whether the attributes specified in specific local locations harbored autocorrelation. Therefore, based on global spatial autocorrelation analysis, local spatial autocorrelation analysis was used to establish the spatial autocorrelation of each township, and make clear the specific spatial location and significance of aggregation. The local indicators of spatial association (LISA) included Moran's I and Getis $G$ statistics, and the most commonly used local Moran's $I$ statistics were applied. Local Moran's $I$ statistic was used to reveal whether the observed variables were clustered in local areas, and the formula is:

$$
I_{i}=\frac{y_{i}-\bar{y}}{\delta^{2}} \sum_{j=1}^{n} W_{i j}\left(y_{j}-\bar{y}\right)
$$

In the formula, $\delta^{2}=\frac{1}{n} \sum_{i=1}^{n}\left(y_{i}-\bar{y}\right)^{2}, \bar{y}=\frac{1}{n} \sum_{i=1}^{n} y_{i}$
Local Moran's $I>0$ indicates that spatial units with similarly observed variables are clustered in space, suggesting that a high-value unit was surrounded by units with similar high value (high-high), or a low-value unit was surrounded by units with similar low value (low-low). This phenomenon is also called "clustering". Moran's $I<0$ indicates that units with dissimilar observed values were clustered in space, and a low value surrounded by a high value (low-high), or a high value surrounded by a low value (high-low). This phenomenon is also called "outlier".

LISA clustering map directly displays aggregation areas and aggregation types. Different colors of small areas represent different local correlation types. Red indicated "high-high" aggregation areas, dark blue indicated "low-low" areas, magenta indicated "highlow" areas, and light blue depicted "low-high" areas [23].

\section{Data processing and statistical analysis}

All data (comprising name, sex, age, lesion type, lesion size, and location) were inputted using the software Epi Info 7.2.4 (Department of Health \& Human Services, USA). Data of 35 counties were subsequently combined. The error and duplicate data were eliminated, whereby the error was corrected via the double-entry comparison method, and error corrections were made using the twoitem comparison method, and eventually a human echinococcosis screening database was created.

Statistical data analyses were executed using the statistical software R 3.0.3 (Lucent Technologies, Jasmine Mountain, USA). We also calculated the prevalence of human echinococcosis in each township.

The prevalence of human echinococcosis was calculated as per the following formula:

$$
P=n / N \times 100 \%
$$

where $P$ is the prevalence of the population in the surveyed area, $n$ refers to the number of patients detected, and $N$ denotes the number of examined people.

ArcGIS 10.3 software for Desktop (Environmental Systems Research Institute, USA) was used to map the spatial distribution map of the human echinococcosis prevalence at the township level and analyze the trend surface, while the GeoDa 1.6.7 software (Center for Spatial Data Science, University of Chicago) was adopted to analyze the prevalence of human echinococcosis in townships for global and local spatial autocorrelations. The spatial autocorrelation indexes were respectively adopted as Global Moran's I index and Anselin's Local Moran's I index, and the related results were visually displayed. A level of $P<0.05$ was considered statistically significant. 


\section{Results}

\section{Basic information}

In total, 649 townships from 35 endemic counties in 4 prefectures/cities of Sichuan Province were recruited to carry out this survey. A total of 2758525 people were examined, and 11743 patients were detected. Notably, the distribution of patients was found in 426 townships, including 405 townships with CE patients, 167 townships with $\mathrm{AE}$ patients, and 146 townships with both kinds of echinococcosis patients (Table 1 ).

Overall, the survey conducted in 2016-2019 demonstrated that the prevalence of $\mathrm{AE}(0.351 \%)$ was significantly higher than that of CE $(0.310 \%)\left(\chi^{2}=28.166\right.$, $P<0.05)$. However, the survey findings of 2012 revealed that the prevalence of CE $(0.881 \%)$ was significantly higher than that of $\mathrm{AE}(0.273 \%)$ [14] $\left(\chi^{2}=372.204\right.$, $P<0.05$, Fig. 2).

\section{Geographical distribution Spatial distribution characteristics}

The prevalence of echinococcosis in Sichuan Province was $0.462 \%$ (see Table 2). Noticeably, 5 out of the 35 endemic counties exhibited a predominance of more than 1\%, including Shiqu (6.512\%), Seda (3.056\%), Baiyu (1.237\%), Dege (1.180\%), and Ganzi (1.171\%) counties.

Based on the analysis at the township level, the top three townships with the highest overall prevalence were all under the jurisdiction of Shiqu county, including Changsha Gongma (14.961\%), Gemeng (12.065\%), and Sexu (10.588\%) townships. Specifically, the top three townships with the highest prevalence of CE were Gayi (3.243\%) and Changxu Gongma (3.210\%) townships in Shiqu county and Suoba (3.220\%) township in Dege county. Moreover, the top three townships with the highest prevalence of AE were Changsha-Gongma (12.986\%), Gemeng (10.462\%), and Mengyi (10.363\%) townships in Shiqu county. From Table 3, the number of townships with human prevalence $0-0.1 \%, 0.1-1 \%$ and $1 \%$ or above was 153,209 , and 64, respectively.

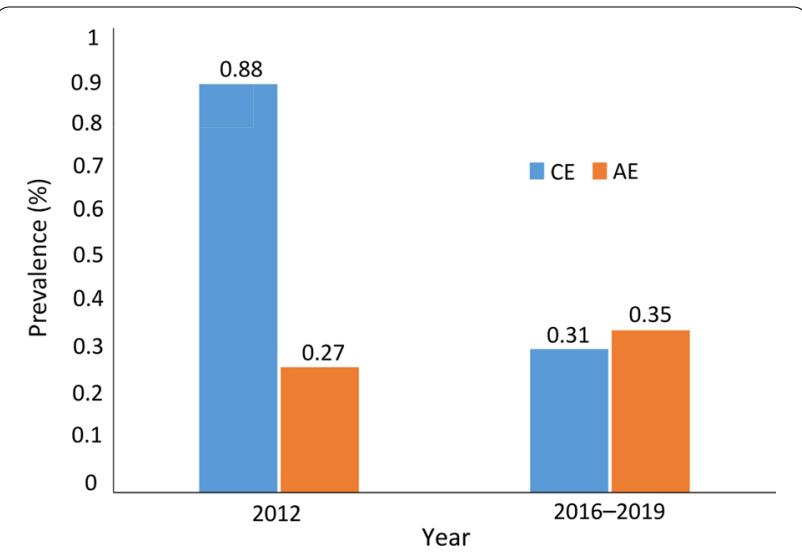

Fig. 2 The comparison of the prevalence of cystic echinococcosis and alveolar echinococcosis in 2012 and 2016-2019. AE alveolar echinococcosis, CE cystic echinococcosis

The spatial distribution map of Sichuan Province displayed that the high prevalence of echinococcosis was primarily distributed in the west, northwest, and north, while the low prevalence was predominantly distributed in the south and east. The prevalence of echinococcosis in Sichuan Province exhibited spatial clustering characteristics, indicating that areas with high or low prevalence seemingly aggregated into pieces. Nevertheless, the judgment of this aggregation was statistically insignificant, hence spatial autocorrelation analysis and other methods were adopted for further inference (Fig. 3).

\section{Spatial clustering analysis}

Global spatial autocorrelation analysis results of echinococcosis prevalence at the township level in Sichuan Province indicated positive spatial autocorrelation and aggregation distribution rather than random distribution (Table 4).

In this subsection, the outcomes of local spatial autocorrelation analysis revealed that the "high-high" gathering areas of overall prevalence were mainly located in the townships of Shiqu, Seda, Ganzi, Dege, and Baiyu counties, totaling 41. The "high-high" gathering areas of

Table 1 Distribution of human echinococcosis at the township level in Sichuan Province

\begin{tabular}{|c|c|c|c|c|}
\hline City/prefecture & $\begin{array}{l}\text { Number of townships } \\
\text { surveyed }\end{array}$ & $\begin{array}{l}\text { Number of CE epidemic } \\
\text { townships }\end{array}$ & $\begin{array}{l}\text { Number of AE epidemic } \\
\text { townships }\end{array}$ & $\begin{array}{l}\text { Number of } \\
\text { total epidemic } \\
\text { townships }\end{array}$ \\
\hline Aba prefecture & 230 & 134 & 40 & 146 \\
\hline Ganzi prefecture & 325 & 255 & 125 & 262 \\
\hline Liangshan Prefecture & 70 & 12 & 0 & 12 \\
\hline Ya'an city & 24 & 4 & 2 & 6 \\
\hline Total & 649 & 405 & 167 & 426 \\
\hline
\end{tabular}

$A E$ alveolar echinococcosis, $C E$ cystic echinococcosis 


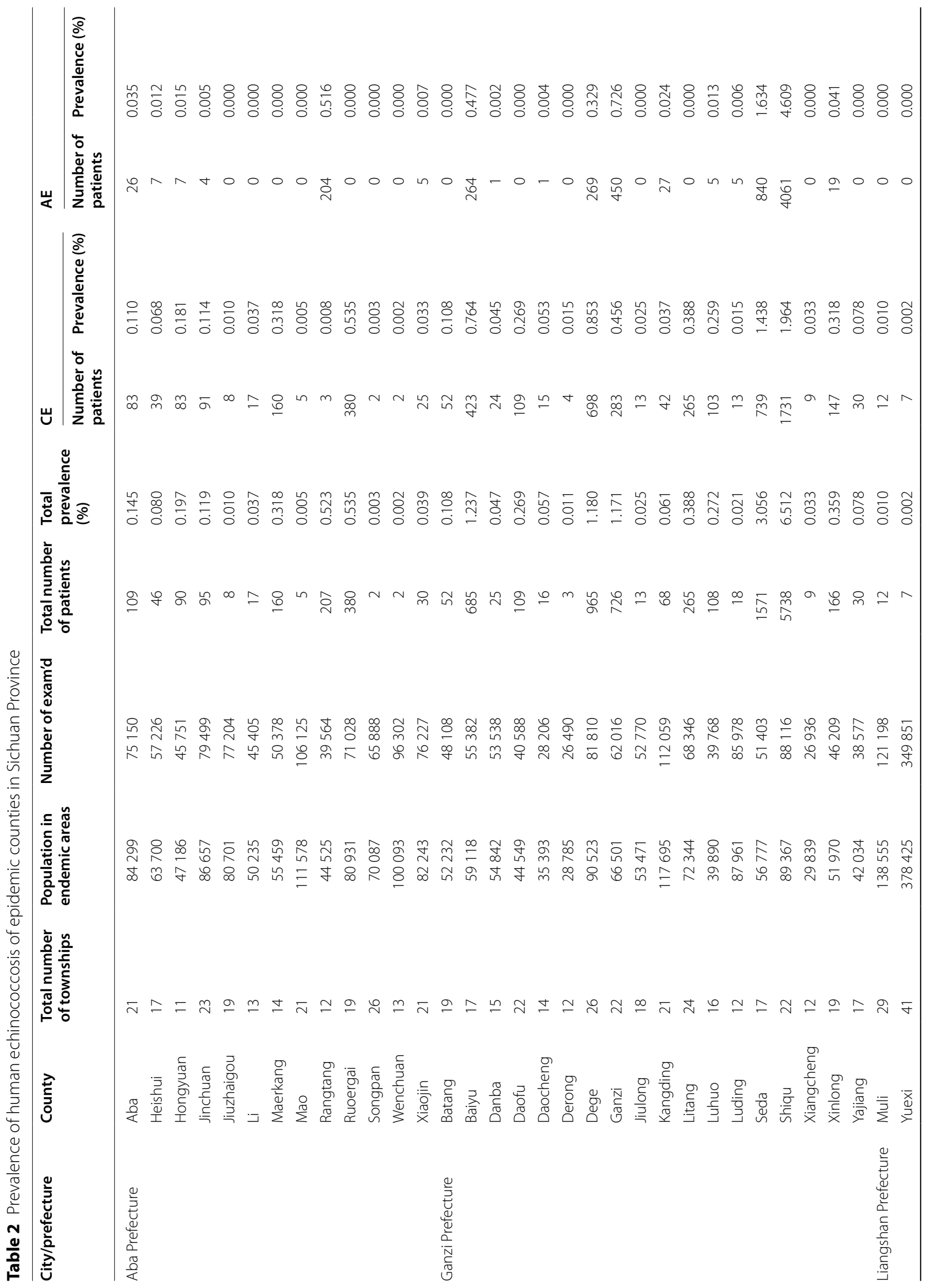


CE prevalence were dominantly located in townships of Shiqu, Seda, Ganzi, Dege, Baiyu, Rangtang, Xinlong, and Litang counties, totaling 48. The "high-high" gathering areas of $\mathrm{AE}$ prevalence were predominantly located in towns of Shiqu, Dege, Seda, Ganzi, and Rangtang counties, totaling 31. The "low-low" gathering areas of the overall prevalence were primarily located in townships of Jiuzhaigou, Songpan, Heishui, Mao, Li, Wenchuan, Xiaojin, Baoxing, Tianquan, Kangding, Luding, Muli, Daocheng, Xiangcheng, and Yuexi counties, totaling 193. The "low-low" gathering areas of the predominance of CE were largely located in townships of Jiuzhaigou, Songpan, Heishui, Mao, Li, Wenchuan, Xiaojin, Baoxing, Tianquan, Kangding, Luding, Muli, Daocheng, Xiangcheng, and Yuexi counties, totaling 165. Eventually, the "low-low" gathering areas of the occurrence of $\mathrm{AE}$ were generally in the townships of Jiuzhaigou, Songpan, and Kangding, totaling 4 (Fig. 4).

\section{Discussion}

Echinococcosis is a global zoonotic parasitic disease that seriously threatens the health and life safety of people. It impacts social and economic development, causing heavy economic burdens to families. It is one of the major reasons for poverty among the population in endemic areas of China [24]. Here, a survey was performed in 35 epidemic counties previously identified in 2012 to understand human echinococcosis prevalence in Sichuan Province. We specifically investigated the epidemic areas and human echinococcosis at the township level in Sichuan Province. Our results reveal that the echinococcosis patients were distributed in 426 townships, primarily located in the northwestern part of Sichuan Province and concentrated in high mountain meadows, pastoral areas with a cold, arid climate, little rainfall, semi-agricultural, and semi-pastoral areas. This is attributed to Echinococcus eggs adapting to the natural environments of cold, dry, and rainless [25, 26]. Additionally, we noted abundant animal resources in these environments, forming a relatively suitable food chain for predation and prey, thus enabling Echinococcus spp. to form a complete life history, resulting in echinococcosis prevalence [27, 28]. $\mathrm{CE}$ and $\mathrm{AE}$ were prevalent in 405 and 167 townships; $\mathrm{CE}$ epidemic areas were more widespread than AE. This observation is potentially attributed to the fact that $\mathrm{CE}$ is primarily spread in the biological circulation chain comprising domestic dogs as the main definitive host and domestic animals as intermediate hosts [29, 30], However, $\mathrm{AE}$ is primarily spread in the biological circulation chain composed of foxes and dogs as the main definitive host and small mammals as intermediate hosts [31]. In contrast, the distribution range of small mammals is smaller than that of domestic animals [32]. 
Based on the results of the 2012 national echinococcosis prevalence survey, the number of patients with $\mathrm{CE}$ in Sichuan Province was distinctively higher than those with AE, the findings of the 2016-2019 survey revealed that $\mathrm{AE}$ patients accounted for $52.77 \%$ of the total number of patients. The major reasons for this discrepancy may be as follows. Firstly, the infectious source of CE was primarily dogs. Besides, since the implementation of the Echinococcosis Prevention and Control Project, Sichuan Province had standardized the management and deworming of dogs, and thus the number of stray dogs reduced; the infectious source was effectively controlled, thereby decreasing the prevalence of echinococcosis [33]. Secondly, the surgical operation difficulty of CE was less compared to that of $\mathrm{AE}$, and more $\mathrm{CE}$ patients were effectively cured [34, 35]. Thirdly, the experience and diagnostic techniques of ultrasonographers differed from one region to another, potentially leading to inaccurate diagnosis.

The predominance of human echinococcosis in Sichuan Province followed a trend in both the east-west and north-south directions for most of the years, gradually decreasing from west to east and from north to south. These findings are consistent with those of trend surface analysis of echinococcosis conducted in Aba Prefecture in 2013 [18] and with the spatial and temporal distribution characteristics of the new human echinococcosis prevalence in Sichuan Province between 2007 and 2017 [36]. The explanations why areas with high prevalence were essentially distributed in the northwest of Sichuan Province may be as follows. Firstly, the northwestern part of Sichuan Province is characterized by the natural environment of high altitude, dry with little rainfall, low temperature, low humidity, low oxygen content, long sunshine time, and most shrub vegetation types are bushes, these factors favor the Echinococcus eggs survival for a longer time, thus exacerbating the spread of echinococcosis [37, 38]. Secondly, studies on the Qinghai-Tibet Plateau in northwest Sichuan Province revealed that ecological changes caused by overgrazing of grassland promoted the spread of multilocular echinococcosis. The overgrazing of grassland decreased the grass density and height, which is beneficial to the habitat formation of small mammals including Microtus fuscus and plateau pika, this significantly increases the number and density of these small mammals. Furthermore, a large number of owned dogs and stray dogs were noted in these areas, this is conducive to the transmission and circulation of $E$. multilocularis, causing a high prevalence of $\mathrm{AE}[39,40]$. Thirdly, a large number of domestic animals as intermediate hosts of $\mathrm{CE}$ increased the spread of echinococcosis, and the grassland was a favorable environment for the intermediate hosts (small mammals) of $\mathrm{AE}[39,41,42]$.
Table 3 Prevalence of different types of echinococcosis in Sichuan Province

\begin{tabular}{llll}
\hline $\begin{array}{l}\text { Human } \\
\text { prevalence } \\
(\%)\end{array}$ & $\begin{array}{l}\text { Number of } \\
\text { CE epidemic } \\
\text { townships }\end{array}$ & $\begin{array}{l}\text { Number of } \\
\text { AE epidemic } \\
\text { townships }\end{array}$ & $\begin{array}{l}\text { Number of } \\
\text { total epidemic } \\
\text { townships }\end{array}$ \\
\hline$\geq 1$ & 47 & 36 & 64 \\
$0.1-1$ & 197 & 71 & 209 \\
$<0.1$ & 161 & 60 & 153 \\
Total & 405 & 167 & 426 \\
\hline
\end{tabular}

$A E$ alveolar echinococcosis, $C E$ cystic echinococcosis

Fourthly, some analysis of risk factors for echinococcosis transmission carried out in Sichuan showed that the large number of dogs per household, the high infection rate of dogs, the high density of dog feces in the household courtyard, the high density of local small mammals, the low awareness rate of prevention and control knowledge in herdsmen, the higher incidence of echinococcosis in aged people, the traditional pastoral nomadic lifestyle, the large numbers of bovine and sheep/goat were all related to the population suffering from echinococcosis $[43,44]$. previous findings showed that the northwestern part of Sichuan Province including Shiqu, Seda, Ganzi, and Baiyu counties had numerous livestock, that could be raised for a long time since local farmers were not willing to kill them. Most areas lacked centralized slaughterhouses or standardized slaughter management, which might be one of the reasons for the higher human prevalence rate in these areas than that in the southeast [45]. Fifthly, many types of wild animals with large quantities were reported in the northwest of Sichuan Province, therefore, snow disasters, lack of enough food in spring, and other reasons caused the death of livestock and wild animals. Consequently, this caused the formation of the circulation chain of echinococcosis transmission, thus aggravating the transmission of echinococcosis [46, 47]. Sixthly, illiteracy among most residents of Tibetan areas in the northwest of Sichuan Province, with the bad production and living habits including close contact with dogs, feeding sick cattle (sheep) viscera to dogs, not washing hands after eating, and lack of safe drinking water sources, significantly increased the risk of transmission infection $[47,48]$.

Numerous reports indicate that the prevalence and transmission of echinococcosis are influenced by natural, biological, and social factors; restricted by the adult worms in definitive host segments and eggs in the external environment; larvae in intermediate hosts and stability of parasites with spatial autocorrelation [35, 49-54].

We employed spatial autocorrelation statistical analysis to explore the spatial distribution characteristics of 
a

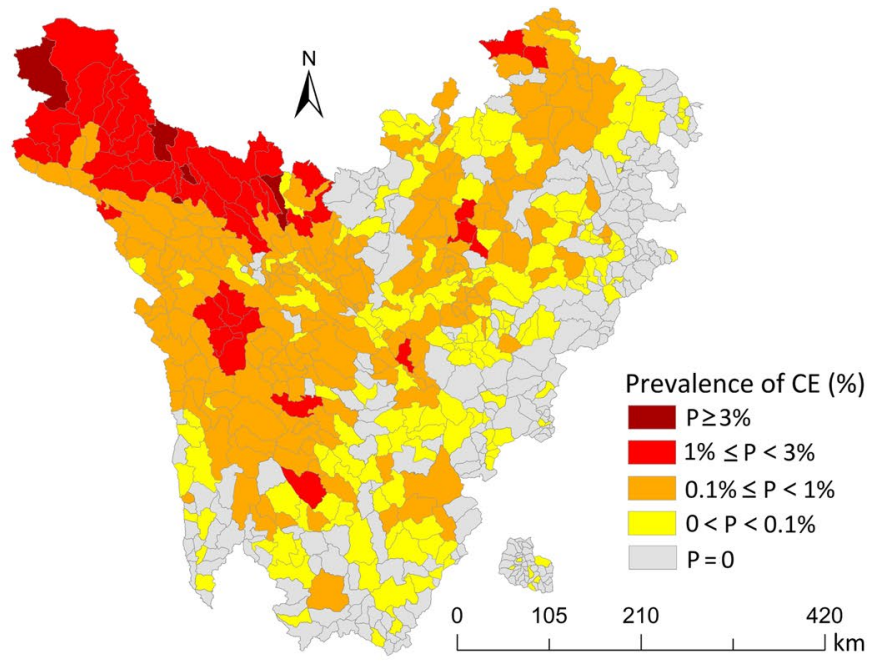

b

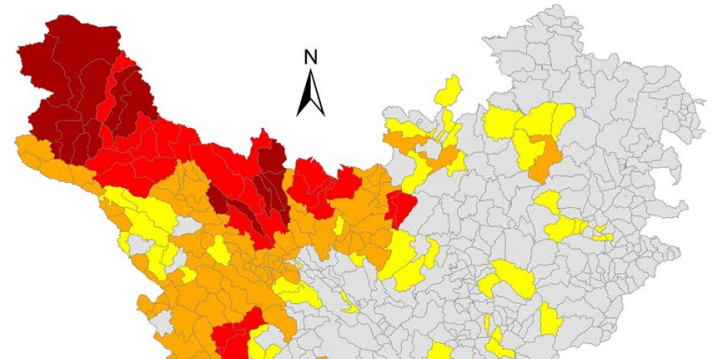

Prevalence of $\mathrm{AE}(\%)$

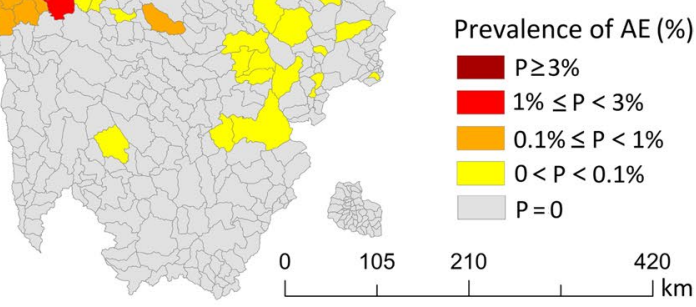

C

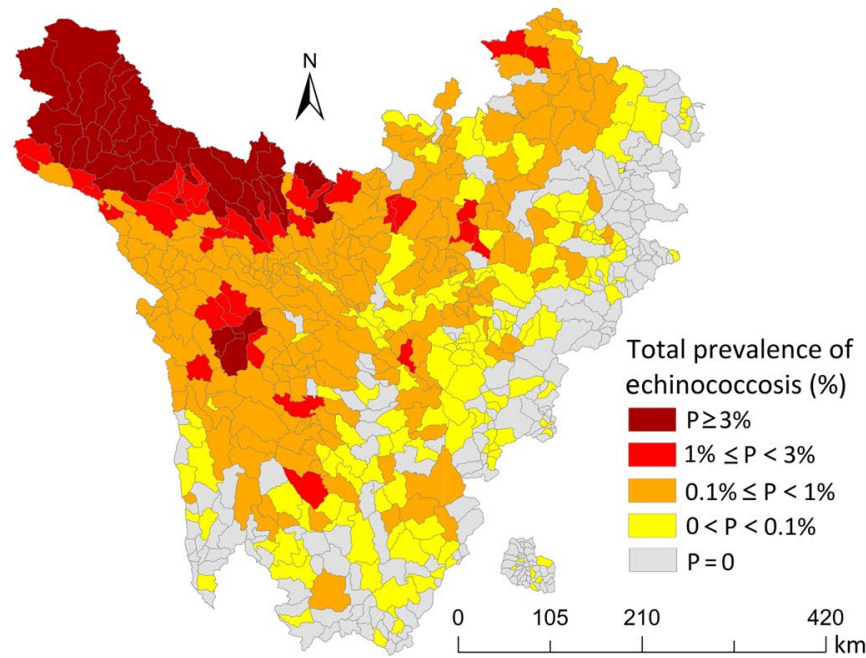

Fig. 3 Distribution of the prevalence of echinococcosis in Sichuan Province. a Distribution of cystic echinococcosis; b Distribution of alveolar echinococcosis; c Distribution of total prevalence of echinococcosis. AE alveolar echinococcosis, CE cystic echinococcosis 
a
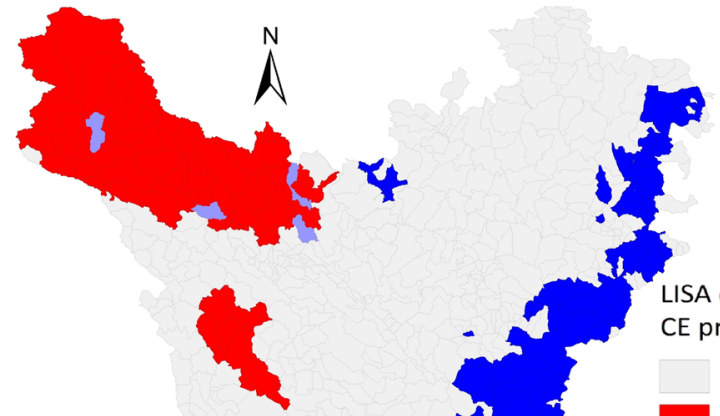

LISA cluster map of

CE prevalence

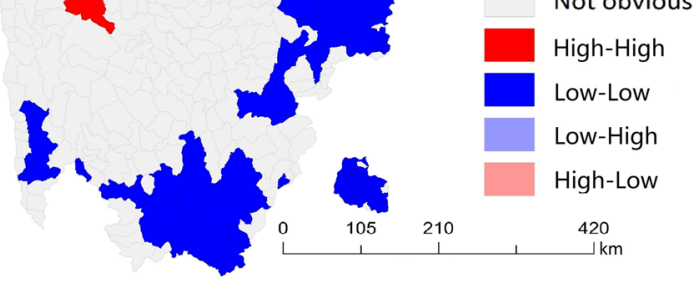

b
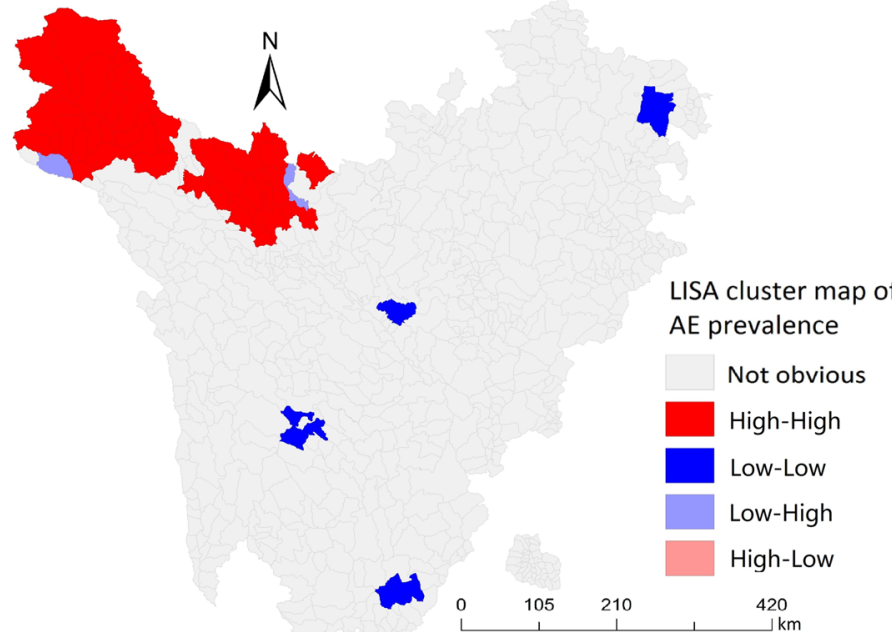

C
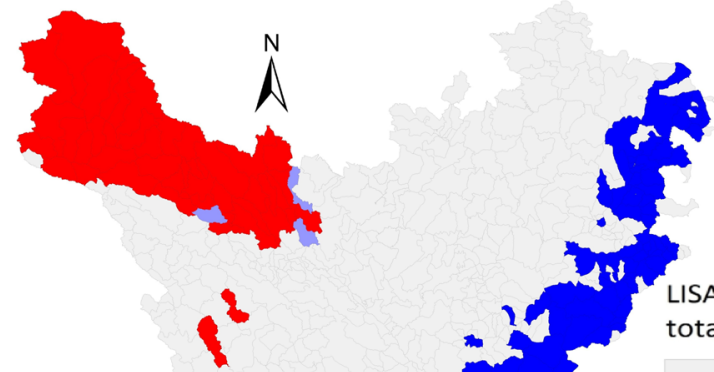

LISA cluster map of total prevalence

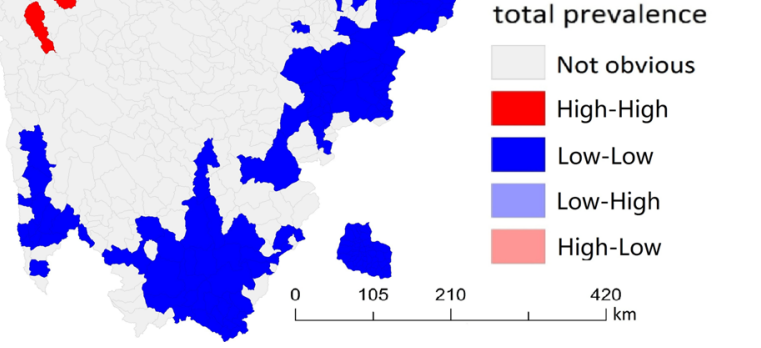

Fig. 4 Local indicators of spatial association (LISA) clustering map of new prevalence of echinococcosis in the rural population of Sichuan Province. a LISA cluster map of CE. $\mathbf{b}$ LISA cluster map of AE. c LISA cluster map of total prevalence of echinococcosis 
Table 4 Spatial autocorrelation global Moran's / analysis on the prevalence of echinococcosis

\begin{tabular}{llll}
\hline $\begin{array}{l}\text { Prevalence of } \\
\text { echinococcosis }\end{array}$ & Moran's I index & Z-value & $\boldsymbol{P}$-value \\
\hline Total & 0.77 & 22.07 & $<0.01$ \\
CE & 0.70 & 29.67 & $<0.01$ \\
AE & 0.71 & 30.52 & $<0.01$ \\
\hline
\end{tabular}

$A E$ alveolar echinococcosis, $C E$ cystic echinococcosis

echinococcosis at the township level in Sichuan Province. Through global autocorrelation analysis of echinococcosis prevalence, and the prevalence of populations CE and $\mathrm{AE}$, the predominance of different types of echinococcosis exhibited a positive spatial correlation, with an aggregated distribution.

Moreover, global spatial autocorrelation analysis results showed that echinococcosis prevalence was clustered in space with a positive spatial correlation. On the other hand, in the local spatial autocorrelation analysis, the LISA aggregation map showed "high-high" and "low-low" clusters of human echinococcosis prevalence at the township level. Notably, the "high-high" clusters were dominantly distributed in most of the townships of Shiqu, Seda, Ganzi, Dege, and Baiyu counties near the northwest of Sichuan Province, this is consistent with the spatial aggregation analysis of echinococcosis conducted in Ganzi Prefecture, Sichuan Province by Zhao et al. [19]. The areas with high prevalence might be linked to special natural environmental and socio-geographical factors influenced by the high altitude. Meanwhile, the unhealthy production lifestyle of local Tibetans might have a significant similarity due to the close geographical location, thereby forming a high-risk behavior of echinococcosis [55]. Since dogs are mobile with a certain range of activities in space, their migration led to spread and prevalence echinococcosis in neighboring areas [56]. Additionally, the "low-low" gathering areas were mostly located in townships of Jiuzhaigou, Songpan, Heishui, Mao, Li, Wenchuan, Xiaojin, Baoxing, Tianquan, Kangding, Luding, Muli, Daocheng, Xiangcheng, and Yuexi counties near the southeast in Sichuan Province. These counties did not belong to the areas with a high prevalence of echinococcosis; their altitude was relatively low; the natural conditions were superior to those of high prevalence areas; production and lifestyle were relatively healthy, hence risks were relatively minimal. These findings suggest the need to strengthen comprehensive prevention and control of echinococcosis in "high-high" gathering areas. Simultaneously, favorable factors for the low prevalence of echinococcosis should be actively explored in "low-low" gathering areas to provide a baseline reference for the prevention and control of echinococcosis in "high-high" gathering areas.

This study has some limitations. Firstly, the human population were screened for hydatid lesions by using portable B-ultrasonography. Only the abdominal lesions of $C E$ and $A E$ could be detected, whereas lesions in the lungs, brain, and other parts outside of the abdomen could not be found. Therefore, the prevalence determined in the survey among people may be lower compared to that of the actual situation. Secondly, this study was carried out in all townships, the findings only reflect the gathering situation at the township level, so the human prevalence at the village level was still unknown. Although studies on limited spatial distribution of echinococcosis have been conducted in Sichuan Province, providing a theoretical and practical reference for control strategy making in the future, more precise spatial aggregation of the prevalence of echinococcosis in Sichuan is needed to verify and explore based on the constantly updated data and spatial analysis at village level.

\section{Conclusions}

This study reported the echinococcosis prevalence and spatial distribution characteristics in human population at the township level in the endemic areas of Sichuan province. Human prevalence of echinococcosis was clustered in space, and the specific clustering areas were identified. Therefore, we suggest to study and formulate different prevention and control strategies in the "highhigh gathering areas" and "low-low gathering areas" in the future, so as to effectively control the epidemic of echinococcosis in Sichuan Province, Thereby reducing the harm of echinococcosis to the people in epidemic areas.

\section{Abbreviations \\ AE: Alveolar echinococcosis; CE: Cystic echinococcosis; DALY: Disability adjusted of life years; LISA: Local indicators of spatial association; PE: Polycystic echinococcosis.}

\section{Acknowledgements}

Thanks to all of the participants for their time and patience in participating in this study.

\section{Authors' contributions}

HW participated in the design and plan of the study and prepared the manuscript for publication. WQ, HY, and ZB helped facilitate and coordinate the study. YWJ, ZGJ, LS, WQ, LRR, YL, DBZL, QSC, WSA and WYX collected the 
data. WLY and LY gave technical assists. All authors read and approved the final manuscript.

\section{Funding}

The research was supported by the Hydatid Disease Control Project of central government transfer payment of China and Sichuan Science and Technology Plan Project (No.2018SZ0116).

\section{Availability of data and materials}

If data is needed, please contact the corresponding author.

\section{Declarations}

\section{Ethics approval and consent to participate}

This survey was approved by the Ethics Committee of Sichuan CDC. Al authors consent to participate under the 'Ethics, consent and permissions' heading. All participants,and signed their own names on the informed consent form before the examination.

\section{Consent for publication}

Not applicable.

\section{Competing interests}

All of the authors declared that they had no competing interests.

\section{Author details}

1Department of Parasitic Diseases, Sichuan Provincial Center for Disease Control and Prevention, No.6 Zhongxue Road, Chengdu 610041, People's Republic of China. ${ }^{2}$ National Institute of Parasitic Diseases, Chinese Centre for Disease Control and Prevention, Chinese Centre for Tropical Diseases Research, WHO Collaborating Centre for Tropical Diseases, National Centre for International Research On Tropical Diseases, Ministry of Science and Technology, NHC Key Laboratory of Parasite and Vector Biology (National Institute of Parasitic Diseases, Chinese Center for Disease Control and Prevention), Shanghai 200025, People's Republic of China. ${ }^{3}$ Doctorate School of Chemical and Biological Sciences for Health (CBS2), University of Montpellier, 34395 Montpellier, France. ${ }^{4}$ Ganzi Prefectural Center for Disease Control and Prevention, No.139 Lucheng South Road, Ganzi Prefecture 626000, People's Republic of China. ${ }^{5}$ Aba Prefectural Center for Disease Control and Prevention, No.178 Meigu Street, Aba Prefecture 624000, People's Republic of China. ${ }^{6}$ Liangshan Prefectural Center for Disease Control and Prevention, Section 2 of Hangtian Avenue, Liangshan Prefecture 615000, People's Republic of China. ${ }^{7}$ Ya'an Prefectural Center for Disease Control and Prevention, No.9 Fangcao Road, Daxing New District, Ya'an City 625000, People's Republic of China.

Received: 29 January 2021 Accepted: 19 May 2021

Published online: 05 June 2021

\section{References}

1. Eckert J, Gemmell MA, Meslin FX, Pawlowski ZS. WHO/OIE manual on echinococcosis in humans and animals: a public health problem of global concern. Vet Parasitol. 2001;31(14):1717-8.

2. Lei ZL, Wang LY. Control situation and primary task of key parasitic diseases in China. Zhongguo Ji Sheng Chong Xue Yu Ji Sheng Chong Bing Za Zhi. 2012;30(1):1-5 (in Chinese).

3. Jenkins DJ, Romig T, Thompson RCA. Emergence/re-emergence of Echinococcus spp._-a global update. Int J Parasit. 2005;35(11-12):1205-19.

4. Thompson RC, McManus DP. Towards a taxonomic revision of the genus Echinococcus. Trend Parasitol. 2002;18(10):452-7.

5. Xiao N, Qiu JM, NaKao M, Li TY, Chen XW, et al. Biological features of a new Echinococcus species (Echinococcus shiquicus) in the east of QinghaiTibet Plateau. Zhongguo Ji Sheng Chong Xue Yu Ji Sheng Chong Bing Za Zhi. 2008;26(4):307-12 (in Chinese).

6. Budke CM, Casulli A, Kern P, Vuitton DA. Cystic and alveolar echinococcosis: successes and continuing challenges. PLoS Neglect Trop Dis. 2017;11(4):54-77.

7. Wen H. Hydatidosis. Beijing: People's Medical Publishing House; 2015 (in Chinese)
8. Brunetti E, Kern P, Vuitton DA. Expert consensus for the diagnosis and treatment of cystic and alveolar echinococcosis in humans. Acta Trop. 2010;114(1):1-16

9. Budke CM, Deplazes P, Torgerson PR. Global socioeconomic impact of cystic echinococcosis. Emerg Infect Dis. 2006;12(2):296-303.

10. Torgerson PR, Macpherson CN. The socioeconomic burden of parasitic zoonoses: global trends. Vet Parasitol. 2011;182(1):79-95.

11. Shi DZ. Geographical distribution of echinococcosis in China. Bull Endemic Dis. 2000;5(1):74-5 (in Chinese).

12. Yang YR, Ellis M, Sun T, Li ZZ, Liu XZ, Vuitton DA, et al. Unique family clustering of human echicoccosis cases in a Chinese community. Am J Trop Med Hyg. 2006;74(3):487-94.

13. Yang W. Epidemic situation and control measures of echinococcosis in Tibetan areas of Sichuan. Proceedings of the National Symposium on Zoonosis. National Symposium on the Prevention and Control of Zoonoses. 2006:2:319-20.

14. Wang GQ. Epidemiological survey on echincoccosis in China. Shanghai: Shanghai Science and Technology Press; 2016 (in Chinese).

15. Waller LA, Gotway CA. Applied spatial statistics for public health data. John Wiley \& Sons; 2004.

16. Liu L, Guo B, Li W, Zhong B, Yang W, Li SC, et al. Geographic distribution of echinococcosis in Tibetan region of Sichuan Province, China. Infect Dis Poverty. 2018;7(1):104-12.

17. Brundu D, Aloi D, Rolesu S, Piseddu T, Masala G, et al. Cystic echinococcosis in slaughtered cattle in Sardinia: a retrospective epidemiological study and spatial analysis. Geospat Health. 2012;6(2):285-91.

18. Qi YF. Study on distribution characteristics and influencing factors of echinococcosis in Aba Prefecture, Sichuan Province. China CDC. 2013. (Master degree thesis in Chinese).

19. Zhao Y, Wu WP, Li W, Xu KJ, Wang LY, Wang Y. Spatial clustering analysis of echinococcosis in Ganzi Tibetan Autonomous Prefecture, Sichuan Province. Int J Med Parasit Dis. 2015;42(3):164-9 (in Chinese).

20. Zheng CJ, Yang L, Zhang GJ, Wang Q, Wu WP, Yan J. Interpretation of technical scheme for echinococcosis control (Edition 2019). Trop Dis Parasit J. 2020;18(4):193-201 (in Chinese).

21. Ministry of Health of the People's Republic of China. Diagnostic criteria for Hydatid Disease (WS 257-2006); 2006 (in Chinese).

22. Li XY, Chen K. Scan statistic theory and its application in spatial epidemiology. Zhonghua Liu Xing Bing Xue Za Zhi. 2008;29(8):828-31 (in Chinese).

23. Zhou XN. Spatial epidemiology. Beijing: Science Press; 2009 (in Chinese).

24. Wang Q, Francis R, Christine B, Philip SC, Xiao YF, Dominique A, et al. Grass height and transmission ecology of Echinococcus multilocularis in Tibetan communities. Chin Med J (Engl). 2010;123(1):61-7.

25. Thompson RCA. Biology and Systematics of Echinococcus. Echinococcus and Echinococcosis. Wallingford: CAB International; 2016.

26. Ishikawa H, Ohga Y, Doi R. A model for the transmission of Echinococcus multilocularis in Hokkaido, Japan. Parasit Res. 2004;91(6):444-51.

27. Zeng XM, Guan YY, Wu WP. Epidemiological distribution characteristics of echinococcosis. Chin J Zoonos. 2014;30(4):413-7 (in Chinese).

28. Yang YR, Clements ACA, Gray DJ, Atkinson JAM, Williams GM, Barnes TS, et al. Impact of anthropogenic and natural environmental changes on Echinococcus transmission in Ningxia Hui Autonomous Region, the People's Republic of China. Parasit Vect. 2012;5(1):146-54.

29. Ziaei H, Fakhar M, Armat S. Epidemiological aspects of cystic echinococcosis in slaughtered herbivores in Sari abattoir, North of Iran. J Parasit Dis. 2011;35(2):215-8.

30. Craig PS, Larrieu E. Control of cystic echinococcosis/hydatidosis: 1863-2002. Adv Parasitol. 2006:61:443-508.

31. Wang Q, Vuitton DA, Xiao YF, Budke CM, Ponce MC, Schantz PM, et al. Pasture types and Echinococcus multilocularis, Tibetan communities. Emerg Infect Dis. 2006;12(6):1008-10.

32. Fang Q. Analysis of epidemic situation and influencing factors of echinococcosis in Qinghai-Tibet Plateau. China CDC. 2014. (Master degree thesis in Chinese).

33. He W, Shang JY, Yu WJ, Zhang GJ, Wang Q, Huang Y, et al. Epidemiological investigation of echinococcosis in shiqu county, Sichuan Province. J Prev Med Inform. 2017;33(9):850-4 (in Chinese).

34. Wu WP, Wang H, Wang Q, Zhou XN, Wang LY, Zheng CJ, et al. A nationwide sampling survey on echinococcosis in China during 2012-2016. 
Zhongguo Ji Sheng Chong Xue Yu Ji Sheng Chong Bing Za Zhi. 2018;36(1):1-14 (in Chinese).

35. Aini A, Shao YM, Aji T, Zhang WB, Lin RY, Hou YY, et al. Establishment and innovative practice of Integrative system of prevention, diagnosis and management for echinococcosis in China. Zhongguo Ji Sheng Chong Xue Yu Ji Sheng Chong Bing Za Zhi. 2019;37(4):388-94 (in Chinese).

36. He W, Liao S, Wang Q, Huang Y, Yu WJ, Zhang GJ, et al. Spatial and temporal distribution of newly diagnosed echinococcosis patients in Sichuan Province from 2007 to 2017. Zhongguo Xue Xi Chong Bing Fang Zhi Za Zhi. 2019;31(4):393-9 (in Chinese).

37. Veit P, Bilger B, Schad V, Schäfer J, Lucius R. Influence of environmental factors on the infectivity of Echinococcus multilocularis eggs. Parasitology. 1995;110(1):79-86.

38. LiTY, Qiu JM, Yang W, Craig PS, Chen XW, Xiao N, et al. Echinococcosis in Tibetan populations, Western Sichuan Province, China. Emerg Infect Dis. 2005;11(12):1866.

39. Wang $Q$, Xiao YF, Dominique AV, Peter MS, Francis R, Christine B, et al. Impact of overgrazing on the transmission of Echinococcus multilocularis in Tibetan pastoral communities of Sichuan Province. China Chin Med J (Engl). 2007;120(3):237-42.

40. Schantz PM, Wang H, Giu J, Liu FJ, Saito E, Emshoff A, et al. Echinococcosis on the Tibetan Plateau: prevalence and risk factors for cystic and alveolar echinococcosis in Tibetan populations in Qinghai Province, China. Parasitology. 2003;127(Suppl7):S109.

41. Fromsa A, Jobre Y. Infection prevalence of hydatidosis (Echinococcus granulosus, Batsch, 1786) in domestic animals in Ethiopia: a synthesis report of previous surveys. Vet J. 2011;15(2):11-33.

42. Ibrahim MM. Study of cystic echinococcosis in slaughtered animals in Al Baha region, Saudi Arabia: interaction between some biotic and abiotic factors. Acta Trop. 2010;113(1):26-33.

43. He W, Wang Q, Huang Y, Yu WJ, Zhang GJ, Liao S, et al. Risk factors of echinococcosis in Shiqu County, Sichuan Province. Zhongguo Ji Sheng Chong Xue Yu Ji Sheng Chong Bing Za Zhi. 2019;37(4):428-32 (in (Chinese).

44. He W, Wang Q, Huang Y, Yu WJ, Zhang GJ, Liao S, et al. Risk factors of human cystic echinococcosis in Shiqu County, Sichuan Province: a case-control study. Zhongguo Xue Xi Chong Bing Fang Zhi Za Zhi. 2019;31(5):486-90 (in Chinese).

45. Guo L, Yang AG, Zhang ZZ, Hou W, Chen D, Wen H, Mao GQ, et al. An epidemiological survey on livestock hydatid diseases in Sichuan Province. Chin J Vet Med. 2012;48(2):25-7 (in Chinese).
46. Wang Q, Vuitton DA, Qiu JM, Giraudoux P, Xiao YF, Schantz PM, Raoul F, et al. Fenced pasture: a possible risk factor for human alveolar echinococcosis in Tibetan pastoralist communities of Sichuan, China. Acta Trop. 2004;90(3):285-93.

47. Wang H. Analysis of human hydatid disease risk factors in Qinghai province. Zhongguo Ji Sheng Chong Xue Yu Ji Sheng Chong Bing Za Zhi. 2004;17(4):214-6 (in Chinese).

48. Qi XW, Feng XH, Freya VK, Li HT, Song T, Duan XY, et al. Epidemic status of Echinococcus granulosus and risk of human cycstic echinococcosis in Hoboksar Mongolian Autonomous County of Xinjiang. Chin J Endemiol. 2010;31(3):297-9 (in Chinese).

49. Miterpáková M, Hurníková Z, Antolová D, Dubinský P. Endoparasites of red Fox (Vulpes vulpes) in the Slovak Republic with the emphasis on zoonotic species Echinococcus multilocularis and Trichinella spp. Helminthologia. 2009;46(2):73-9.

50. Gudewar J, Pan D, Bera K, Das K, Konar J, Rao A, et al. Molecular characterization of Echinococcus granulosus of Indian animal isolates on the basis of nuclear and mitochondrial genotype. Mol Biol Rep. 2009;36:1381-5.

51. Let H, Pearson MS, Blair D. Complete mitochondrial genomes confirm the distinctiveness of the horse-dog and sheep-dog strains of Echinococcus granulosus. Parasitology. 2002;124(1):97-112.

52. Capuano F, Rinaldi L, Maurelli MP, Perugini AG, Veneziano V, Garippa G, et al. Cystic echinococcosis in water buffaloes: epidemiological survey and molecular evidence of ovine (G1) and Buffalo (G3) strains. Vet Parasitol. 2006;137(3-4):262-8.

53. Yamamoto N, Kishi R, Katakura Y, Miyake H. Risk factors for human alveolar echinococcosis: a case-control study in Hokkaido. Japan Ann Trop Med Parasitol. 2001;95(7):689-96.

54. Zhao Y, Wu WP. Application of spatial statistics on echinococcosis studies. Chin J Zoonos. 2015;31 (3):272-6 (in Chinese).

55. Gongsang QZ, Wang LY, Niu YL, Baima YJ, Ciren LM, Xiao D, et al. Analysis of the characteristics of the spatial distribution of human echinococciasis in the Tibet Autonomous Region. J Parasit Biol. 2018;13(1):64-7.

56. Zeng XM, Wang LY, Wu WP, Guan YY, Fang Q. Cluster analysis of cystic echinococcosis in non Tibetan Plateau regions. Zhongguo Xue Xi Chong Bing Fang Zhi Za Zhi. 2014;2:180-3 (in Chinese).
Ready to submit your research? Choose BMC and benefit from:

- fast, convenient online submission

- thorough peer review by experienced researchers in your field

- rapid publication on acceptance

- support for research data, including large and complex data types

- gold Open Access which fosters wider collaboration and increased citations

- maximum visibility for your research: over 100M website views per year

At BMC, research is always in progress.

Learn more biomedcentral.com/submissions 